\title{
Updated and revised neutron reaction data for ${ }^{237} \mathrm{~Np}$
}

\author{
Guochang Chen ${ }^{1, a}$, Jimin Wang ${ }^{1}$, Wentian $\mathrm{Cao}^{2}$, Guoyou Tang ${ }^{2}$, and Baosheng $\mathrm{Yu}^{1}$ \\ 1 China Nuclear Data Center, China Institute of Atomic Energy, Beijing 102413, China \\ 2 School of Physics \& State Key Laboratory of Nuclear Physics and Technology, Peking University, Beijing 100871, China
}

\begin{abstract}
Nuclear data with high accuracy for minor actinides play an important role in nuclear technology applications, including reactor design and operation, fuel cycle, estimation of the amount of minor actinides in high burn-up reactors and the minor actinides transmutation. Based on the evaluated experimental data, the updated and revised evaluation of a full set of $n+{ }^{237} \mathrm{~Np}$ nuclear data from $10^{-5} \mathrm{eV} \sim 20 \mathrm{MeV}$ are carried out and recommended. Mainly revised quantities are neutron multiplicities from fission reaction, inelastic, fission, $(\mathrm{n}, 2 \mathrm{n})$ and $(\mathrm{n}, \gamma)$ reaction cross sections as well as angular distribution and so on. The promising results are obtained when the renewal evaluated data of ${ }^{237} \mathrm{~Np}$ will be used to instead of the evaluated data in CENDL-3.1 database.
\end{abstract}

\section{Introduction}

Nuclear data with high accuracy for minor actinides (MAs) are required to estimate the amount of minor actinides in high burn-up reactors and to research a technology to transmute MAs to short half-lived nuclides or stable ones. In fission reactors, the mainly generated MAs are ${ }^{237,239} \mathrm{~Np},{ }^{238} \mathrm{Pu},{ }^{241,243} \mathrm{Am},{ }^{242,244} \mathrm{Cm}$ and so on. A calculation of H.Takano [1] shows that the amount of ${ }^{237} \mathrm{~Np}$ is about $4.40 \%$ of total amount of generated MAs and that of ${ }^{241} \mathrm{Am} 58.24 \%$ in a $\mathrm{UO}_{2}-\mathrm{PWR}$, and that ${ }^{237} \mathrm{~Np}$ is $50.71 \%$ and ${ }^{241} \mathrm{Am} 34.55 \%$ in a MOX-PWR in $50 \mathrm{GWd} / \mathrm{t}$ burn-up and 7 years cooling condition.

The data of ${ }^{237} \mathrm{~Np}$ in CENDL-3.1 [2] were revised in 2003, and there exist many new evaluations [3-6] and measurements on it, and the standard reaction cross sections [5] were updated in 2008. In the present work the data for ${ }^{237} \mathrm{~Np}$ in CENDL-3.1 [2] are revised. Mainly revised are neutron multiplicities from fission reaction, inelastic, $(n, 2 n)$ and $(n, f)$ reaction cross sections as well as angular distributions and so on.

\section{Evaluation procedure and results}

\subsection{Evaluation procedure}

There is a significant amount of measurements with different methods, facilities and neutron sources available for neutron reactions on ${ }^{237} \mathrm{~Np}$ in present study. Most of the experimental data are adopted from the EXFOR/CINDA database [7], the Data Bank of the Nuclear Energy Agency in Paris, INIS database and relevant periodical literatures. There exist more or less discrepancy in those experimental data for the same neutron reaction. For cross sections evaluation, a primary effort is made in the experimental data analysis and evaluation, which includes systematic accumulation, correction, evaluation of all relevant experimental data, and re-normalization of the neutron data to neutron cross section standards [5] and ${ }^{252} \mathrm{Cf}$ neutron multiplicities from spontaneous fission, etc.

In order to fit the measurements for total cross section, nonelastic scattering cross section and elastic scattering angular distribution, the neutron optical model parameters (OMP) are obtained with the APMN code [8], which is a program for searching automatically an optimum set of neutron optical model parameters. The theoretical models are adopted as the coupled-channel optical model ECIS-95 [9] code and Hauser-Feshbach statistical plus pre-equilibrium theory FUNF [10] code in present work. The sequence usually followed in the evaluation above resonance region is to optimize the agreement of model calculated results with the evaluated experimental data for cross sections, differential cross sections and double differential cross sections, by careful model parameter adjustment. After that the experimental data after evaluation and theoretical results are assembled into formal evaluated nuclear data files with ENDF format and make fine modifications generally within experimental uncertainties to enhance the agreement with simple fast critical benchmark measurements.

\subsection{Evaluation of nubar}

Most of the measurements for prompt neutron multiplicities from fission reaction $\left(v_{p}\right)$ are relevant to "standard" or other accurately measured reactions. Most of the $v_{p}$ measurements are relative to ${ }^{252} \mathrm{Cf}$ neutron multiplicities from spontaneous fission, which is very accurately known as $3.7692 \pm 0.0047[11,12]$. For $v_{p}$ measurements are corrected to consist with IAEA recommendation $[11,12]$. The available experimental data and systematics are used in nubar evaluation also. According to the dependence of $v_{p}$ on incident neutron energy is given by linear function below $20 \mathrm{MeV}$ as Function (1), which is fit to the evaluated experimental data.

$$
v_{p}=2.59+0.15 \times E_{n}
$$

(C) The Authors, published by EDP Sciences. This is an Open Access article distributed under the terms of the Creative Commons Attribution License 4.0 (http://creativecommons.org/licenses/by/4.0/). 


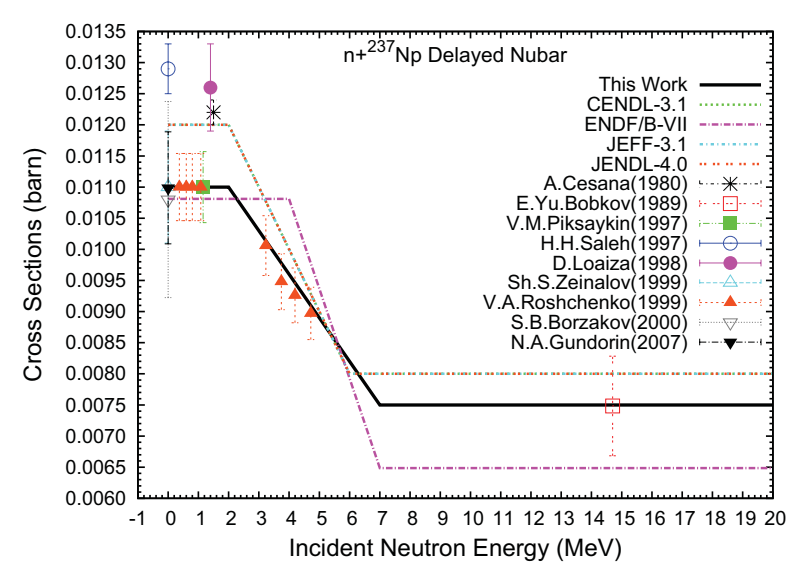

Figure 1. The evaluated delayed fission neutron multiplicity, $v_{d}$, compared with the measured and other evaluated data.

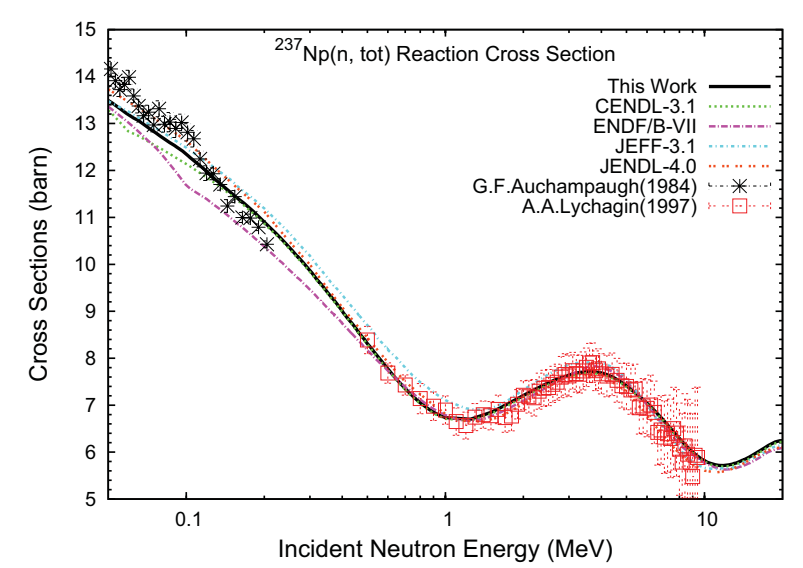

Figure 2. Comparison of (n, tot) reaction between the evaluations and measurements.

For delayed neutron multiplicity per fission $\left(v_{d}\right)$, there have some measurements [13-21]. According to analysis measurements, V.M. Piksaykin [15] is the same group as V.A. Roshchenko [19] and normalized to its of V.A.Roshchenko [19], and the data of S.B. Borzakov [20] and Sh.S. Zeinalov [18] are normalized to its of N.A.Gundorin [21] that they are the same group of Dubna. The present result of $v_{d}$ is obtained by fitting the evaluated measurements as shown in Fig. 1, which agree well with the experimental data after analysis and evaluation. However, the evaluation data between different evaluation databases exist discrepancy in the whole energy range. The total average neutron multiplicity per fission (total nubar) is a sum of average delayed $\left(v_{d}\right)$ and prompt $\left(v_{p}\right)$ neutron multiplicity per fission.

\subsection{Optical model parameters}

Based on the available measurements of total, nonelastic scattering and elastic scattering angular distribution above $100 \mathrm{keV}$, a new set of OMP is obtained with slightly adjustment of CENDL-3.1 OMP. The comparison of total cross section is shown in Fig. 2 between the evaluations and measurements. In general, present result agree well with the measurements [22,23] and JENDL-4.0 [4], and it is higher than ENDF/B-VII [5] below $0.6 \mathrm{MeV}$ and lower than JEFF-3.1 [6] below $2 \mathrm{MeV}$.

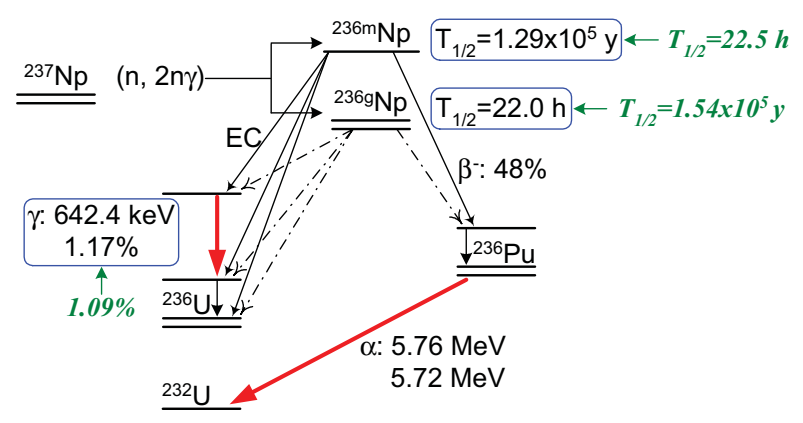

Figure 3. Simplified reaction and decay scheme of ${ }^{237} \mathrm{~Np}(\mathrm{n}, 2 \mathrm{n})$ reaction (measured transitions are indicated as red arrow line).

\section{4. ( $(n, 2 n)$ reaction cross section}

Many measurements [24-30] have been reported on $(\mathrm{n}, 2 \mathrm{n})$ reaction before 1985 . After then, no experimental data is available. According to the accumulated measurements, this reaction produces the meta-stable state of ${ }^{236} \mathrm{~Np}$ as well as its ground state. Principally, three methods to determine the cross section are taken into account in measurement, which are pointed out in the simplified reaction process and decay scheme as shown in Fig. 3: (1) the measurement of prompt $\gamma$ rays accompanying the neutron emission as ${ }^{237} \mathrm{~Np}(\mathrm{n}, 2 \mathrm{n} \gamma)$ reaction process, (2) the direct measurement of $\gamma$ rays following the electron capture (EC) of ${ }^{236 m} \mathrm{~Np}$ to ${ }^{236} \mathrm{U}$, and (3) the measurement of $\alpha$ particles of the ${ }^{236} \mathrm{Pu}$ decay which is obtained by $\beta^{-}$decay of ${ }^{236 m} \mathrm{~Np}$. Method (1) and (2) could not be performed successfully or obtained promising results as the high background radiation disturbs the detection of $\gamma$ rays from $(n, 2 n)$ reaction. It should take notice of the decay information in the reaction process. In Fig. 3, the decay information in the square frame is directly taken from the Refs. [24-30] and the ENSDF evaluation decay data [31] are adopted and shown after the arrow line.

According to the recently decay information of ${ }^{236} \mathrm{~Np}$, these measurements [24-30] actually measure ${ }^{237} \mathrm{~Np}(\mathrm{n}, 2 \mathrm{n})^{236 m} \mathrm{~Np}$ reaction. Therefore, The isomer ratio is primarily important data to estimate the higher MAs production and evaluate the available measurements. In the present work, the isomer ratio (ratio of ${ }^{236 m} \mathrm{~Np}$ production to total $(\mathrm{n}, 2 \mathrm{n})$ cross section) is assumed as $74 \%$ on the basis of experimental data [25,26,32], which is used to correct the experimental data of ${ }^{237} \mathrm{~Np}(\mathrm{n}$, $2 n)^{236 m} \mathrm{~Np}$ reaction cross sections. The present result is obtained by fitting the experimental data after analysis and evaluation. The comparison of $(n, 2 n)$ reaction cross section is shown in Fig. 4 between the evaluations and the measurements after correction. The present result is fit well with measurements in the whole energy range. There exist big discrepancy between different evaluations at $10 \sim 14 \mathrm{MeV}$ and above $15 \mathrm{MeV}$ neutron energy range for missing experimental data.

\subsection{Fission cross section}

There are many experimental data of the fission cross section in the energy range above $100 \mathrm{keV}$, which are measured using relevant to the standard reaction cross section such as ${ }^{235,238} \mathrm{U}(\mathrm{n}, \mathrm{f})$ or other accurately measured reactions such as ${ }^{239} \mathrm{Pu}(\mathrm{n}, \mathrm{f})$. The discrepancy of measurements from different laboratories above $100 \mathrm{keV}$ 


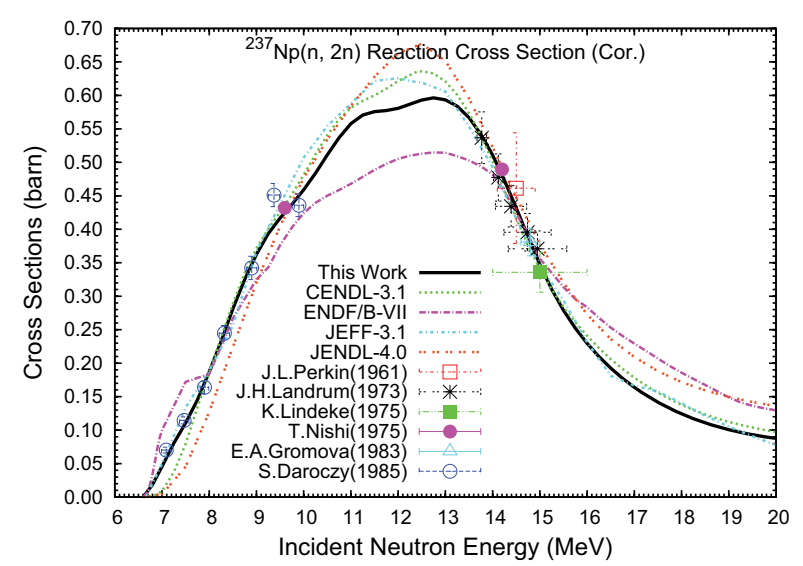

Figure 4. Comparison of (n, $2 n$ ) reaction between the evaluations and evaluated measurements.

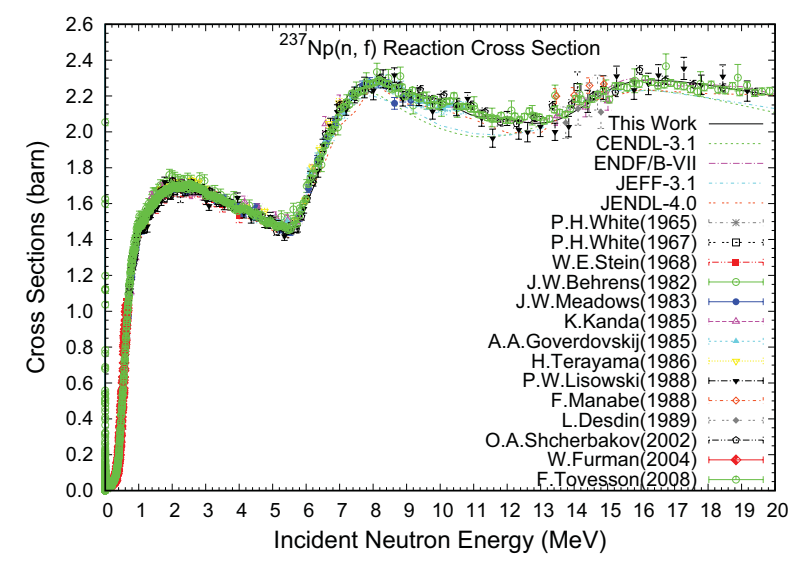

Figure 5. Comparison of (n, f) reaction between the evaluations and measurements.

is about $10 \sim 30 \%$ before 1975 . After then, the fission cross section ratios between ${ }^{237} \mathrm{~Np}$ and ${ }^{235} \mathrm{U}$ are measured by different laboratories [33-40] below $20 \mathrm{MeV}$ using the so-called "threshold cross section method". Their data are consistent with each other within the uncertainty. This is a significant improvement for reducing either the experimental uncertainty or the discrepancy. The above measurements are re-normalized to neutron cross section standards [5].

The data are measured by J.W. Behrens [34] and J.W. Meadows [35] with "threshold cross section method" are important data and give more weight. And the measurement of J.W. Meadows [35] is regarded as the foundation around $14 \mathrm{MeV}$. The fission cross sections between 0.577 and $196 \mathrm{MeV}$ are measured by O.A. Shcherbakov [36] using the TOF method with spallation neutron source. The measurements of F. Tovesson [39,40] are spread around the accuracy ones as the results belong to the process report 2007 and 2008. These measurements are the important experimental information in present work.

The fission cross section is obtained by fitting the measurements after correction. The present result is compared with the measurements and other evaluations as shown in Fig. 5. The measurements are agree well with each other after analysis, evaluation and re-normalization to standard reaction cross section. The present evaluation consistent with ENDF/B-VII [5], JENDL-4.0 [4] and the measurements very well.

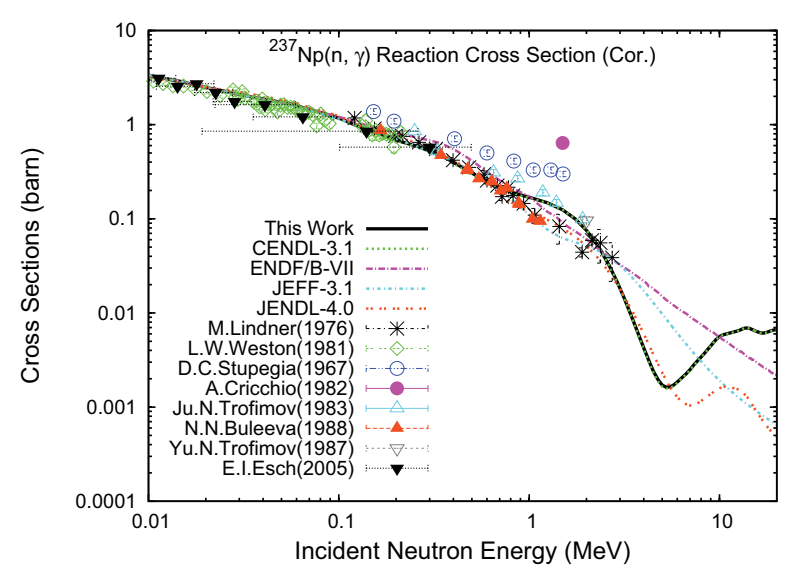

Figure 6. Comparison of the evaluated and experimental data for $(\mathrm{n}, \gamma)$ reaction.

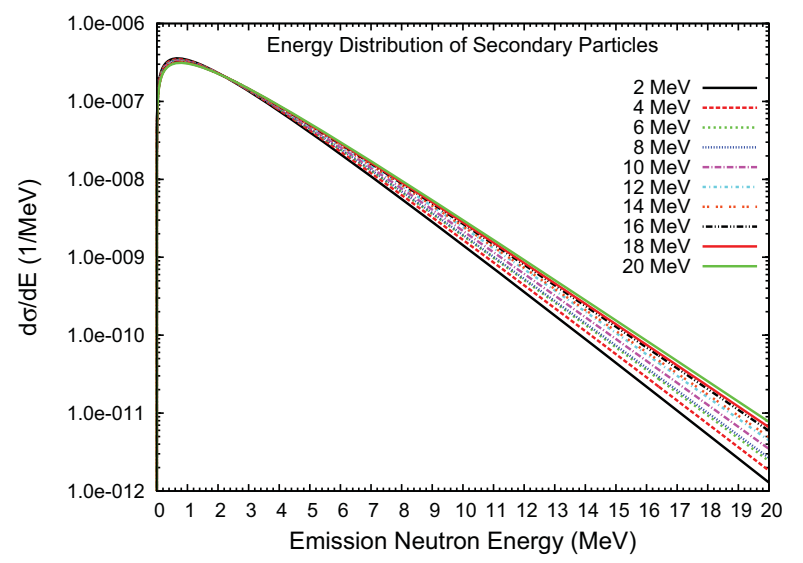

Figure 7. Comparison of neutron spectra for ${ }^{237} \mathrm{~Np}(\mathrm{n}, \mathrm{f})$ reaction.

\subsection{Radiation capture cross section}

For radiation capture reaction, some measurements are available from thermal to $2.7 \mathrm{MeV}$, and some of its [41-45] reported in resonance energy region after 2000. For radiation capture reaction measurements are corrected in decay information and standard reaction cross section. Based on the theoretical calculation and experimental data, the evaluated data is obtained, which reproduce the experimental data very well as shown in Fig. 6. In general, the present result is agree well with the measurements, and each evaluation is consistent well below $2 \mathrm{MeV}$. However, there exist discrepancy between different evaluations above $2 \mathrm{MeV}$ for missing experimental data.

\subsection{Differential cross sections}

The FUNF theoretical calculated result are adopted for the angular distribution and energy spectra. The comparison of the neutron emission spectra for fission reaction is shown in Fig. 7 with different incident neutron energy.

\section{Conclusions}

A full set of $n+{ }^{237} \mathrm{~Np}$ neutron reaction data from $10^{-5} \mathrm{eV} \sim 20 \mathrm{MeV}$ is updated and revised. The present evaluation is compared with other evaluations [2,4-6] and measurements. From the point of view of the microscopic data, the present result is much better than the data in CENDL-3.1 [2], and reproduces the latest experimental 
data very well. And the promising results of ${ }^{237} \mathrm{~Np}$ will be used to instead of the evaluated data in CENDL-3.1 [2].

\section{References}

[1] H. Takano, K. Nishihara, K. Tsujimoto, et al., Prog. Nucl. Energy 37, 371 (2000)

[2] Ge Z.G., Zhuang Y.X, Liu T.J., et al., J. Korean Physical Society 59, 1052 (2011)

[3] Evaluation of neutron nuclear data for ROSFOND2010 (2009)

[4] Keiichi Shibata, Osamu Iwamoto, et al., Jou. Nucl. Sci. Tech. 48, 1 (2011)

[5] M.B. Chadwick, M. Herman, P. Obložinský, et al., Nucl. Data Sheets 112, 2887 (2011)

[6] OECD/NEA Data Bank, JEFF Report 22, OECD/NEA Data Bank, [S. 1.]: [s.n.] (2009)

[7] N. Otuka, et al., Nucl. Data Sheets 120, 272 (2014)

[8] Shen Qingbiao, APOM94 code, Private Communication, 2003

[9] J. Raynal, CEA-N-2772 (1994)

[10] Zhang Jingshang, CNIC-01782 (2005)

[11] A.L. Nichols, et al., INDC(NDS)-0502 (2007)

[12] A.L. Nichols, et al., INDC(NDS)-0534 (2008)

[13] A. Cesana, et al., INDC(NDS)-113, 33 (1980)

[14] E.Yu. Bobkov, A.N. Gudkov et al., Journ. Atomnaya Energiya 67(6), 408 (1989)

[15] V.M. Piksaykin, Yu.F. Balakshev et al., Conf. on Nucl. Data for Sci. and Techn. (Trieste) 1, 485 (1997)

[16] H.H. Saleh, T.A. Parish et al., Journ. Nuclear Science and Engineering, 125, 51 (1997)

[17] D. Loaiza, G. Brunson et al., Journ. Nuclear Science and Engineering, 128, 270 (1998)

[18] Sh.S. Zeinalov, O.V. Zeinalova et al., Conf. Report: Joint Inst. for Nucl. Res., 212(98), 348 (1999)

[19] V.A. Roshchenko, V.M. Piksaykin et al., Physics of Atomic Nuclei 62(8), 1279 (1999)

[20] S.B. Borzakov, et al., Yadernaya Fizika 63, 589 (2000)

[21] N.A. Gundorin, et al., Yadernaya Fizika 70, 1011 (2007)
[22] G.F. Auchampaugh, M.S. Moore, J.D. Moses, et al., Physical Review C 29, 174 (1984)

[23] A.A. Lychagin, B.V. Zhuravlev, V.I. Trykova, et al., S,JINR-E3-97-213, 355 (1997)

[24] J. Perkin et al., Nucl. ENERG. 14, 69 (1961)

[25] J. Landrum et al., Phys. Rev. C8, 1938 (1973)

[26] K. Lindeke, Phys. Rev. C12, 1507 (1975)

[27] Nishi et al., P, INDC(JAP)-28L, 20 (1975)

[28] E. Fort et al., 1982 ANTWERP, 673 (1982)

[29] E.A. Gromova et al., J, Atom. Energy 54, 108 (1983)

[30] S. Daroczy et al., J, Atom. Energy 58(2), 117 (1985)

[31] E. Browne, J.K. Tuli, Nucl. Data Sheets 107, 2649 (2006)

[32] W.A. Myers, M. Lindner, et al., Journal of Inorganic and Nuclear Chemistry 37(3), 637 (1975)

[33] A.D. Carlson, B.H. Patrick, Conf.on Nucl.Cross Sections F.Techn., 971 (1979)

[34] J.W. Behrens, et al., Nucl. Sci. and Engineering 80, 393 (1982)

[35] J.W. Meadows, Nucl. Sci. Engineering 85, 271 (1983)

[36] O.A. Shcherbakov, A.Yu. Donets et al., Conf. Interaction of Neutrons with Nuclei, 257 (2001) or Jou. Nucl. Sci. Tech. 2(1), 230 (2002)

[37] W. Furman, P. Cennini et al., Conf. on Nucl. Data for Sci. and Techn., 1039 (2004)

[38] C. Paradela, L. Audouin et al., Conf. Advances in Nucl.Analysis and Simul., B076 (2006)

[39] F. Tovesson, et al., Phys. Rev. C 75, 034610 (2007)

[40] F. Tovesson, et al., Nucl. Sci. Engineering 195, 94 (2008)

[41] Katsuhei Kobayashi, Samyol Lee, et al., Joul. of Nuclear Science and Technology 39(2), 111 (2002)

[42] T. Katoh, S. Nakamura et al., Joul. of Nuclear Science and Technology 40, 559 (2003)

[43] O. Shcherbakov, K. Furutaka et al., Joul. of Nuclear Science and Technology 42(2), 135 (2005)

[44] C. Guerrero, U. Abbondanno et al., Conf. Advances in Nucl. Analysis and Simul., C031 (2006)

[45] E.I. Esch, et al., Physical Review C 77, 034309 (2008) 